\title{
Drift Compression of Space-Charge-Dominated Bunched Beams*
}

\author{
Hong Qin, Chang Jun, Ronald C. Davidson, and Philip Heitzenroeder \\ Princeton Plasma Physics Laboratory, Princeton University, Princeton, NJ 08543
}

\begin{abstract}
The longitudinal dynamics of drift compression is studied for a space-charge-dominated beam is studied. A nonperiodic quadrupole lattice is designed for a beam undergoing drift compression, and an adiabatically-matched solution is found for the transverse envelope equations in the non-peroidic lattice.
\end{abstract}

\section{INTRODUCTION}

In the currently envisioned configurations for heavy ion fusion (HIF), it is necessary to longitudinally compress the beam bunches by a large factor after the acceleration phase and before the beam particles are focused onto the fusion target. In order to obtain enough fusion energy gain, the peak current for each beam is required to be order $10^{3} \mathrm{~A}$, and the bunch length to be as short as $0.5 \mathrm{~m}$. However, to deliver the beam particles at the required energy, it is both expensive and technically difficult to accelerate short bunches at high current. First of all, because of the finite rise time of the accelerating waveform, it is much easier to accelerate and transport beam bunches longer than $10 \mathrm{~m}$. Second, short bunches have higher current (density) and therefore stronger space-charge effects which can increase the beam emittance and induce halo particles.

The objective of drift compression is to compress a long beam bunch by imposing a negative longitudinal velocity tilt over the length of the beam in the beam frame. As a result, the beam length is reduced as the beam drifts downstream, until the space-charge force in the longitudinal direction becomes strong enough to remove the initial velocity tilt. Different longitudinal compression schemes have been studied in particle simulations $[1,2]$ and in numerical solutions of a cold-fluid model $[3,4,5]$. There are also variations in the assumed axial line density profile of the bunched beam. In Haber's study [1], for example, the line density is uniform in the middle of the pulse but falls off at the ends, whereas in the study of Bisognano, et al [3], the line density is parabolic. Not all compression schemes can be rigorously called drift compression in the sense that in some studies a potential is continuously applied to the beam [1], or several midcourse pulse reshapings are needed [4]. In this paper, we first study the longitudinal dynamics of drift compression using a longitudinal envelope equation derived from an exact self-similar solution of a one-dimensional warm-fluid model. Because the space-charge force increases as the beam is compressed, a larger focusing force is needed to confine the beam in the transverse direction. It is necessary to have a non-

\footnotetext{
* Research supported by the U.S. Department of Energy.
}

periodic quadrupole lattice along the beam path when the beam is undergoing longitudinal compression. In this paper, we describe the design of such a focusing lattice, in which we search for "adiabatically"-matched solutions of the transverse envelope equations. Because the lattice is non-periodic, the concept of a "matched" beam is not welldefined. However, if the non-periodicity is relatively weak, we can still attempt to find "adiabatically"-matched solutions. We will use the following set of beam parameters typical of heavy ion fusion in the present study, while the general methods are applicable to other parameter regimes and application areas. We consider a $\mathrm{Cs}^{+}$beam with rest mass $m=133 m_{p}$, where $m_{p}$ is the proton mass, kinetic energy $(\gamma-1) m c^{2}=2.5 \mathrm{GeV}$, and initial beam length $z_{b 0}=9.5 \mathrm{~m}$. Our goal is to compress the beam by a factor of 16 , i.e. , $z_{b f}=z_{b 0} / 16=0.6 \mathrm{~m}$. The final average current is taken to be $\left\langle I_{f}\right\rangle=2500 \mathrm{~A}$.

\section{LONGITUDINAL DYNAMICS}

We use a one-dimensional warm-fluid model to describe the longitudinal dynamics of drift compression. For the longitudinal electric field, the conventional $g$-factor model is adopted with:

$$
e E_{z}=-\frac{g e^{2}}{\gamma^{2}} \frac{\partial \lambda}{\partial z}, \quad g=1+2 \ln \frac{r_{w}}{r_{b}} .
$$

Here, $e$ is the charge, $\lambda(t, z)$ is the line density, $r_{w}$ is the wall radius, and $r_{b}$ is the average beam radius. We also allow for an externally applied focusing force $F_{z}=-\kappa_{z} z$. In the beam frame, the warm-fluid equations for the line density $\lambda(t, z)$, longitudinal velocity $v_{z}(t, z)$, and longitudinal pressure $p_{z}(t, z)$ are given by

$$
\begin{gathered}
\frac{\partial \lambda}{\partial t}+\frac{\partial}{\partial z}\left(\lambda v_{z}\right)=0 \\
\frac{\partial v_{z}}{\partial t}+v_{z} \frac{\partial v_{z}}{\partial z}+\frac{e^{2} g}{m \gamma^{5}} \frac{\partial \lambda}{\partial z}+\frac{\kappa_{z} z}{m \gamma^{3}}+\frac{r_{b}^{2}}{m \gamma^{3} \lambda} \frac{\partial p_{z}}{\partial z}=0 \\
\frac{\partial p_{z}}{\partial t}+v_{z} \frac{\partial p_{z}}{\partial z}+3 p_{z} \frac{\partial v_{z}}{\partial z}=0
\end{gathered}
$$

We treat $g$ and $r_{b}$ as constants for present purposes. Equations. (2) , (3) and (4) form a nonlinear hyperbolic system of partial differential equations (PDEs). If $\kappa_{z}$ and $p_{z}$ are neglected, Eqs. (2) and (3) have the same form as the shallowwater equations. Equation (4) is a simplified version of the energy equation derived by Lund and Davidson[6], and it can be recast into the form $\left(\frac{\partial v_{z}}{\partial t}+v_{z} \frac{\partial v_{z}}{\partial z}\right)\left(p_{z} / \lambda^{3}\right)=$ 
0. There exists at least one self-similar solution of the nonlinear hyperbolic PDE system (2), (3) and (4). We let

$$
\begin{aligned}
\lambda(t, z) & =\lambda_{b}(t)\left(1-\frac{z^{2}}{z_{b}^{2}(t)}\right), \\
v_{z}(t, z) & =-v_{z b}(t) \frac{z}{z_{b}(t)}, \\
p_{z}(t, z) & =p_{z b}(t)\left(1-\frac{z^{2}}{z_{b}^{2}(t)}\right)^{2}, \\
\frac{d z_{b}(t)}{d t} & =-v_{z b}(t) .
\end{aligned}
$$

Substituting Eqs. (5), (6) and (7) into Eqs. (2) and (4), we find that the $z$-dependence drops out, and

$$
\begin{aligned}
\frac{d \lambda_{b}}{d t}-\frac{v_{z b}}{z_{b}} \lambda_{b} & =0, \\
\frac{d p_{z b}}{d t}-3 \frac{v_{z b}}{z_{b}} p_{z b} & =0 .
\end{aligned}
$$

Remarkably, but not surprisingly, for the momentum equation (3), the $z$-dependence also drops out, giving

$$
-\frac{d v_{z b}}{d t}-\frac{e^{2} g}{m \gamma^{5}} \frac{2 \lambda_{b}}{z_{b}}+\frac{\kappa_{z} z_{b}}{m \gamma^{3}}-\frac{4 r_{b}^{2} p_{z b}}{m \gamma^{3} \lambda_{b} z_{b}}=0
$$

In general, Eqs. (8) - (11) form a coupled ordinary differential equation system. Most remarkably, these equations recover the longitudinal envelope equation [7]. From Eqs. (8), (10), and (9), we obtain

$$
\begin{aligned}
& \frac{1}{\lambda_{b}} \frac{d \lambda_{b}}{d t}+\frac{1}{z_{b}} \frac{d z_{b}}{d t}=0 \Longrightarrow z_{b} \lambda_{b}=\text { const. }=\frac{3}{4} N_{b}, \\
& \frac{1}{p_{z b}} \frac{d p_{z b}}{d t}+\frac{3}{z_{b}} \frac{d z_{b}}{d t}=0 \Longrightarrow z_{b}^{3} p_{z b}=\text { const. }=W
\end{aligned}
$$

where $N_{b}$ is total number of particles in the bunch. Equation (13) implies that $p_{z}$ increases as $z_{b}$ decreases. The energy equation (4) predicts that the charge bunch is heated in the longitudinal direction when it is compressed. Substituting Eqs. (12), (13) and (8) into Eq. (11), we obtain

$$
\frac{d^{2} z_{b}}{d s^{2}}+\frac{\kappa_{z}}{m \gamma^{3} \beta^{2} c^{2}} z_{b}-K_{l} \frac{1}{z_{b}^{2}}-\varepsilon_{l}^{2} \frac{1}{z_{b}^{3}}=0
$$

where $s=\beta c t, K_{l} \equiv 3 N_{b} e^{2} g / 2 m \gamma^{5} \beta^{2} c^{2}$ is the effective longitudinal self-field perveance, and $\varepsilon_{l} \equiv$ $\left(4 r_{b}^{2} W / m \gamma^{3} \beta^{2} c^{2}\right)^{1 / 2}$ is the longitudinal emittance. Equation (14) is the familiar longitudinal envelope equation. Finally, the self-similar solutions (5), (6) and (7) can be obtained by solving the longitudinal envelope equation (14) numerically.

For $\kappa_{z}=0$, the longitudinal envelope equation (14) can be integrated once to give the relation

$$
\left(z_{b 0}^{\prime 2}-z_{b f}^{\prime 2}\right)=2 K_{l}\left(\frac{1}{z_{b f}}-\frac{1}{z_{b 0}}\right)+\varepsilon_{l}^{2}\left(\frac{1}{z_{b f}^{2}}-\frac{1}{z_{b 0}^{2}}\right),
$$

where $z_{b 0}=z_{b}(s=0), z_{b f}=z_{b}\left(s=s_{f}\right), z_{b 0}^{\prime}=$ $d z_{b} / d s(s=0)$, and $z_{b f}^{\prime}=d z_{b} / d s\left(s=s_{f}\right)$. For given values of $\left(z_{b f}, z_{b 0}, K_{l}, \varepsilon_{l}\right)$, it is desirable to minimize the velocity tilt (momentum spread) $\left(z_{b f}^{\prime}, z_{b 0}^{\prime}\right)$ and the beam path length $s_{f}$. However, $z_{b f}^{\prime}, z_{b 0}^{\prime}$, and $s_{f}$ cannot be chosen arbitrarily because of the constraint imposed by the energy relation (15). From Eq. (14), the beam path length required for drift compression can be expressed as

$$
s_{f}=-\int_{z_{b 0}}^{z_{b f}} \frac{d z_{b}}{\sqrt{z_{b 0}^{\prime 2}-2 K_{l}\left(\frac{1}{z_{b}}-\frac{1}{z_{b 0}}\right)-\varepsilon_{l}^{2}\left(\frac{1}{z_{b}^{2}}-\frac{1}{z_{b 0}^{2}}\right)}} .
$$

In the drift compression scheme considered in this paper, the longitudinal emittance is taken to be $\varepsilon_{l}=7.7 \times 10^{-6}$ $\mathrm{m}$, and $K_{l}=1.3 \times 10^{-4} \mathrm{~m}$, corresponding to an average final current $\left\langle I_{f}\right\rangle=2500 \mathrm{~A}, z_{b f}=0.6 \mathrm{~m}$, and $g=2.0$. Assuming $z_{b 0}^{\prime}=-0.023$, we obtain $s_{f}=421.5 \mathrm{~m}$ by evaluating the integral in Eq. (16), and $z_{b f}^{\prime}=-0.01$ by solving Eq. (15). The axial beam size $z_{b}(s)$, obtained numerically from the longitudinal envelope equation (14), is plotted together with the velocity tilt $z_{b}^{\prime}(s)$ in Fig. 1.
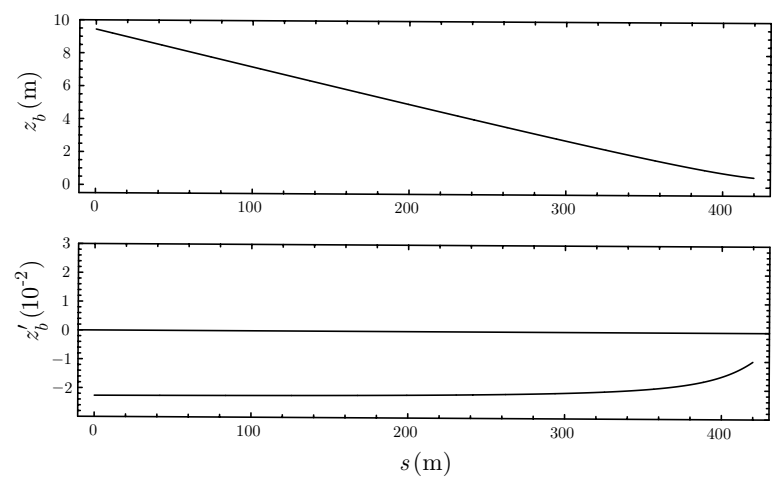

Figure 1: Longitudinal drift compression of a heavy ion fusion beam.

\section{TRANSVERSE DYNAMICS}

For a long charge bunch, the transverse dynamics in a quadrupole lattice is described approximately by the transverse envelope equations:

$$
\begin{aligned}
& \frac{d^{2} a}{d s^{2}}+\kappa_{q} a-\frac{2 K(s)}{a+b}-\frac{\varepsilon_{x}^{2}}{a^{3}}=0 \\
& \frac{d^{2} b}{d s^{2}}-\kappa_{q} b-\frac{2 K(s)}{a+b}-\frac{\varepsilon_{y}^{2}}{b^{3}}=0
\end{aligned}
$$

where $K(s) \equiv 2 N_{b} e^{2} / m \gamma^{3} \beta^{2} c^{2} z_{b}(s)$ is the effective perveance. Because $K(s)$ is an increasing function of $s$, it is necessary to increase the strength of the quadrupole lattice coefficient $\kappa_{q}(s)$ along the beam path to reduce the expansion of the beam radius. Since the quadrupole lattice is not periodic, the concept of a "matched" beam is not well defined. However, if the the non-periodicity is small, that is, if the quadrupole lattice changes slowly along the beam path, we can seek an "adiabatically"-matched beam which, by definition, is locally matched everywhere. For a given slowly changing non-periodic lattice, we assume 

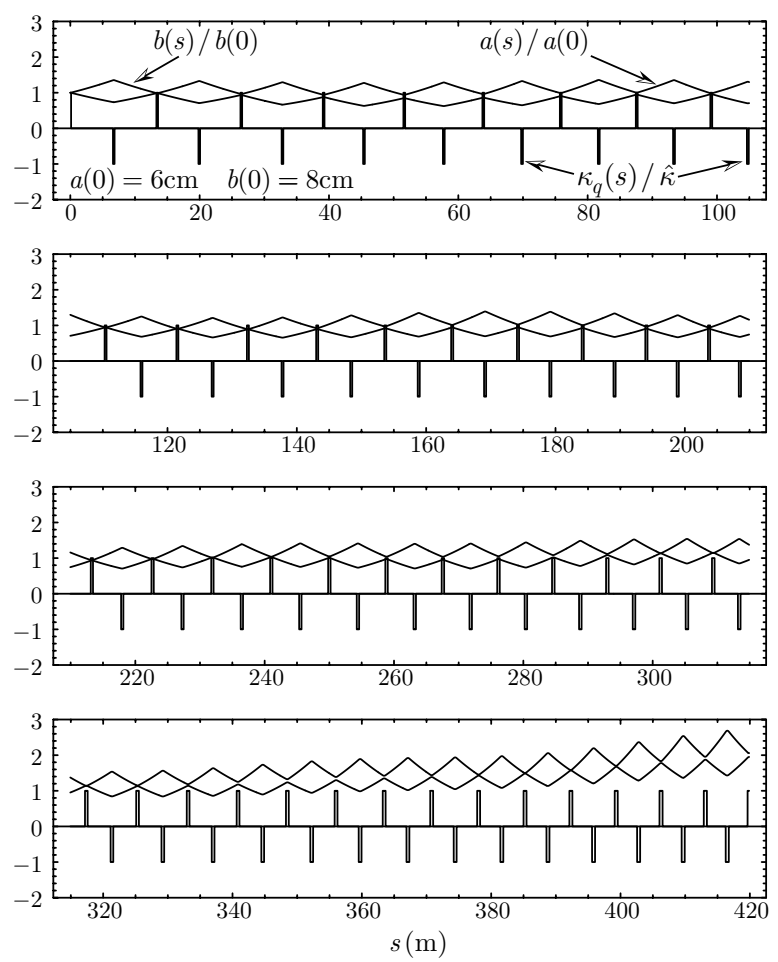

Figure 2: Adiabatically-matched envelope solutions in a non-periodic lattice for a heavy ion fusion beam under drift compression.

such an adiabatically-matched solution indeed exists. On the other hand, for the problem of drift compression, we describe the design of a non-periodic lattice which provides the required control of beam radius when the beam is compressed, and equally importantly, minimizes the possibility of global mismatch. It is intuitive that a lattice, which keeps both the vacuum phase advance and depressed phase advance constant, is less likely to induce beam mismatch. Lee, et al [8], derived the expressions for the vacuum phase advance $\sigma_{v}$ and depressed phase advance $\sigma$ given by

$$
\begin{aligned}
2\left(1-\cos \sigma_{v}\right) & =\left(1-\frac{2 \eta}{3}\right) \eta^{2}\left(\frac{B^{\prime}}{[B \rho]}\right)^{2} L^{4}, \\
\sigma^{2} & =2\left(1-\cos \sigma_{v}\right)-K\left(\frac{2 L}{\langle a\rangle}\right)^{2} .
\end{aligned}
$$

Here, $\eta$ is the filling factor, $L$ is the lattice period, $B^{\prime}$ is field gradient of the magnets, and $\langle a\rangle$ is the average beam radius. Assuming $\eta \ll 1$, we obtain

$$
\eta^{2}\left(\frac{B^{\prime}}{[B \rho]}\right)^{2} L^{4}=\text { const., } K\left(\frac{2 L}{\langle a\rangle}\right)^{2}=\text { const. },
$$

for constant vacuum phase advance and constant depressed phase advance. For the drift compression scheme considered here, $K_{f} / K_{0}=16$. If we allow $\langle a\rangle$ to increase by a factor of 2, i.e., $\langle a\rangle_{f} /\langle a\rangle_{0}=2$, we obtain $L_{f} / L_{0}=1 / 2$, and $\left(\eta B^{\prime}\right)_{f} /\left(\eta B^{\prime}\right)_{0}=4$. We determine $K(s)$ from the solution of the longitudinal envelope equation. The value of $\langle a\rangle$ is determined from the solutions to Eqs. (17) and (18). For the lattice design, we need to specify $\eta, B^{\prime}$, and $L$. If we choose $L_{i}=L_{0} \exp \left(-s_{i} / s_{f} \ln 2\right)$, and $B_{i}^{\prime}=$ const., then from Eq. (21), $\eta_{i}=\eta_{0} \exp \left(s_{i} / s_{f} \ln 2\right)$, where $s_{i}=$ $\sum_{j=0}^{i-1} L_{j}$. We also choose self-consistently the following system parameters: $\sigma_{v}=72^{\circ}, B_{i}^{\prime}=31.70 \mathrm{~T} / \mathrm{m}, L_{0}=$ $6.72 \mathrm{~m}$, and $\eta_{0}=0.036$. The focusing strength of each magnet is $\widehat{\kappa}=0.38 \mathrm{~m}^{-2}$. Let $N$ denote the total number of quadrupole magnet sets. From $s_{f}=\sum_{j=0}^{N-1} L_{j}$, we obtain $N=45$. The lattice design is illustrated in Fig. 2 together with the solutions to Eqs. (17) and (18). After determining the non-periodic lattice layout, we search iteratively for the adiabatically-matched solutions to Eqs. (17) and (18). Currently, there are no well-defined rules to determine $a$ prior which solution is adiabatically-matched. In general, satisfactory results can be obtained by using an intuitive trial-and-error approach. The solutions plotted in Fig. 2 are adiabatically-matched because the envelope is locally matched and contains no oscillations other than the local envelope oscillations. On the global scale, the beam radius increases monotonically. From the numerical solution shown in Fig. 2, the average beam size increases by a factor of 2 , which agrees with the design assumption.

\section{CONCLUSIONS AND FUTURE WORK}

In this paper, we have studied the longitudinal dynamics of drift compression for a space-charge-dominated beam using a longitudinal envelope equation which describes the self-similar drift compression solution of a onedimensional warm-fluid model. A non-periodic quadrupole lattice configuration has been designed for a beam undergoing drift compression with fixed vacuum phase advance and depressed phase advance. An adiabatically-matched solution was found for the transverse envelope equations in the non-periodic lattice. Research in this area is still far from complete. Many important questions remain to be answered. In particular, physics issues related to the stability of drift compression schemes, longitudinal "shock" formation, and emittance growth during the compression deserve special attention. New results in these areas will be reported in the future.

\section{REFERENCES}

[1] I. Haber, Proc. of the Symposium on Accelerator. Aspects of HIF, GSI-82-8, Darmstadt, W. Germany, 372 (1982).

[2] I. Hofmann and Bozsik, ibid, 362.

[3] J. Bisognano, et al, Laser Program Annual Report 84, LLNL, 3-28 (1985).

[4] D. D.-M. Ho, et al, Particle Accel. 35, 15(1991).

[5] M. J. L. de Hoon, Ph.D. Dissertation, UC Berkeley (2001).

[6] S. M. Lund and R. C. Davidson, Phys. Plasmas 5, 3028 (1998).

[7] D. Neuffer, Particle Accel. 11, 23 (1980).

[8] E. P. Lee, et al, LBL Report 19560 (1985). 